\title{
Initial Growth of GaN and InN Over GaAs (110) Substrates
}

\author{
A. de Paiva, J. L. A. Alves, and H. W. Leite Alves \\ Departamento de Ciências Naturais, Universidade Federal de São João del Rei \\ Caixa Postal 110, 36.300-000, São João del Rei, MG, Brazil
}

Received on 31 March, 2003

\begin{abstract}
We present, a systematic theoretical study of the adsorption of $\mathrm{Ga}$, In and $\mathrm{N}$ over GaAs (110) surfaces based on parameter-free, self-consistent total energy and force calculations using the density functional theory. We analyzed the changes in the bond-lengths and in the bond-angles before and after deposition, as well as the total energy behaviour with the adsorbate chemical potential variation. Our results show that the GaN growth over GaAs (110) is energetically more favorable than the InN growth. However, the resulting $\mathrm{InN}$ film can be cubic only if we apply small strains on the substrate, while the $\mathrm{GaN}$ one is hexagonal.
\end{abstract}

\section{Introduction}

The group III-nitrides(AlN, GaN, InN) and the corresponding alloys have attracted great interest due to their succesful applications in the electronic and optoelectronic device technology [1]. However, their growth in the zincblende structure has been a hard task to the experimentalists, once the most stable structure for these compounds is the wurtzite one.

To solve this interesting puzzle, a lot of substrates has been proposed for the III-nitride growth nitride growth in the cubic modification, and the nitridation of the GaAs surfaces seems to be the most efficient method for such growth [2-4].

In order to understand the physical mechanisms of this new growth technique, we present, in this work, a systematic theoretical study of the adsorption of $\mathrm{Ga}$, In and $\mathrm{N}$ over GaAs (110) surfaces based on the density functional theory, the local-density approximation for the exchangecorrelation term, within the plane-wave pseudopotential method (abinit code [5]). We have used the HartwigsenGoedecker-Hutter ultrasoft pseudopotentials [6]. The surfaces were described by slab supercells build up of 7 atomic layers and a vacuum region equivalent of 5 atomic layers. Also, a (4 4 2) Monkhorst-Pack set of $\mathbf{k}$-points was used to sample the slab Brillouin zone [7]. Details about the convergence of the equilibrium bulk properties of GaAs, as well as the relaxed GaAs (110) surface properties were described in our previous work [8].

\section{$2 \mathrm{~N}$ adsorption}

In Fig. 2, we show the variation of the total energy of the slab as a function of the height $\Delta z$ (in $\AA$ ) of the $\mathrm{N}$ atom(referenced to to uppermost As atom) over the GaAs (110) surface, at the sites defined in Fig. 1 (the drawn lines a)
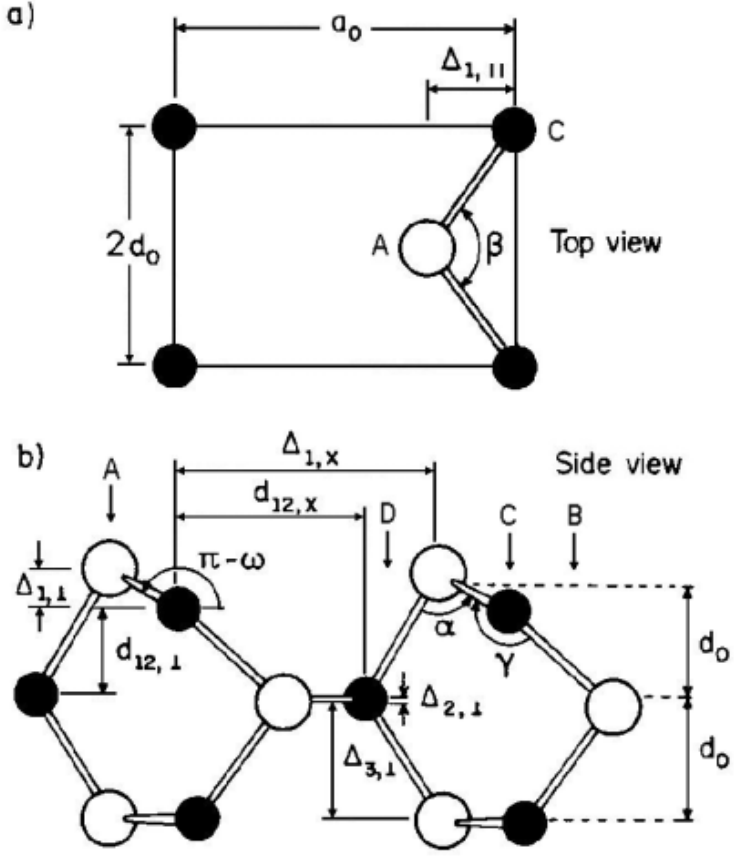

Figure 1. Atomic geometry of the GaAs (110) surfaces. (a) Top view of the surface unit cell. (b) Side view of the first three layers of the (110) surface. Open circles are anions and the shaded ones are cations. $a_{0}$ is the lattice parameter, $d_{0}=\sqrt{ } 2\left(a_{0} / 4\right)$, and the letters $\mathrm{A}, \mathrm{B}, \mathrm{C}, \mathrm{D}$ indicate the possible $\mathrm{N}$ adsorption sites.

are guides to the eyes). For the adsorption of $\mathrm{Ga}$ and In atoms, our results show the same trends. From Fig. 2, the N atom can be adsorbed at GaAs (110) surface only at the A, $\mathrm{B}$ and $\mathrm{D}$ sites. However, at the A and D sites, the final state seems to be a metastable one, and the $\mathrm{N}$ atom needs to overcome an energy barrier of $\sim 0.5-1.2 \mathrm{eV}$ (to arrive at the sites A and $\mathrm{D}$, respectively). So, the most stable site for the $\mathrm{N}$ adsorption over GaAs (110) surface is the B one. To reach this site, $\mathrm{N}$ will overcome an energy barrier of $200 \mathrm{meV}$ and the adsorption energy is $216 \mathrm{meV} / \mathrm{atom}$, in consonance with the experimental conditions of the plasma nitridation 
[4]. For the Ga and In adsorptions, we found the same features, and the calculated adsorption energies are 303 and 389 meV/atom, respectively.

It is interesting to note, from Fig. 2, that the stable position the adsorbed $\mathrm{N}$ atom will be $0.25 \AA$ below the As surface atom. Based on this fact, we, then, have simulated the surface relaxation with the adsorbed $\mathrm{N}$ atom at the $\mathrm{B}$ site, and the final result is depicted in Fig. 3.

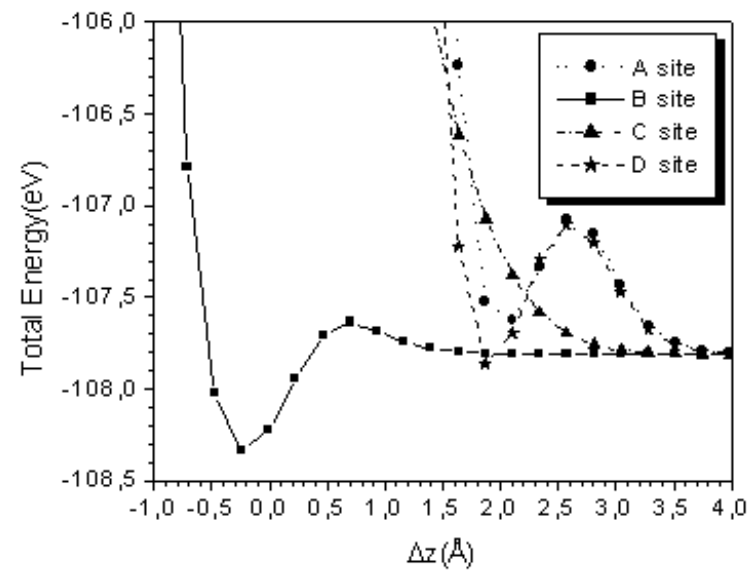

Figure 2. Variation of the total energy of the slab supercell as a function of the height $\Delta \mathrm{z}$ (in $\AA$ ) of the $\mathrm{N}$ atom over the GaAs (110) surface at the sites A, B, C, and D, as defined in Fig. 1.
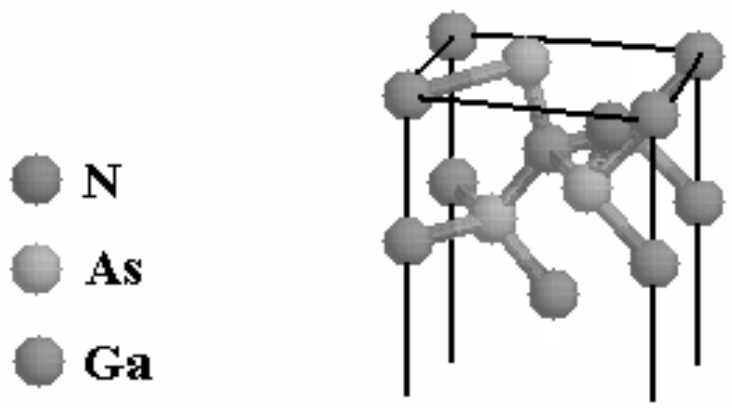

Figure 3. Relaxed atomic positions after the $\mathrm{N}$ adsorption at the GaAs (110) surface.

We can infer, from Fig. 3, that the presence of $\mathrm{N}$ atom pushes both the uppermost $\mathrm{Ga}$ and As atom back to the surface, creating an interesting metastable state: The $\mathrm{N}$ binds to both the uppermost $\mathrm{Ga}$ atom and the As in the sub-surface layer. At the same time, the $\mathrm{N}$ attracts the uppermost As atom, stretching the surface $\mathrm{Ga}-\mathrm{As}$ bonds. As the $\mathrm{N}$ atom is more electronegative than As one and, as it arrives at the surface with sufficiently high energy due to the $\mathrm{N}$ plasma created in the nitridation, the adsorption process finishes by the break the surface Ga-As bonds, and the $\mathrm{N}$ atoms, then, can replace the surface As ones by a simple anion exchange. This should be the mechanism of the As mediated growth for $\mathrm{GaN}$ over GaAs substrates with a surfactant aspect [9].

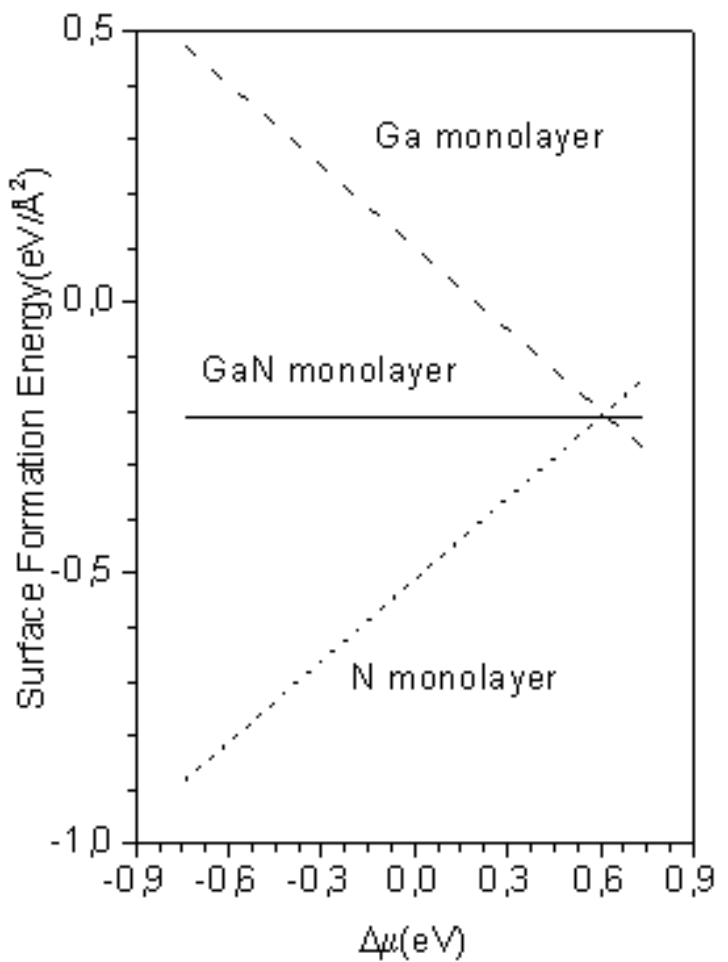

Figure 4. Surface formation energy, $\gamma$, of a N, Ga and GaN monolayers over GaAs (110) surface, as a function of the difference of the chemical potentials of the monolayer constituents, $\Delta \mu$ for neutral charge states.

\section{GaN and InN monolayer adsorp- tion}

We also simulated the adsorption of a monolayer of GaN, and of InN, over GaAs (110) surface. Our calculated results for some structural parameters are depicted in Table I, compared with those of $\mathrm{GaN}$ and $\mathrm{InN}$ (110) surfaces [10]. First, we note that, in both cases, the uppermost layer(the $\mathrm{GaN}$ or InN monolayer) moves inward the surface, but it still keeps the fact that the cation relaxes inward and the anion outward [8]. Moreover, the final value for the tilt angle $\omega$ is less than that observed for the $\mathrm{GaN}$ and $\mathrm{InN}$, and the GaAs layers does not relaxe too much from their original positions. Then, we can infer from our results, that the final GaN or InN thin films growth on GaAs (110) surfaces will not preserve the final (110) structure. The observed strains on the interface with the substrate suggest that, from Table I, the Nitride film structure changes toward a hexagonal one, as already observed experimentally [4]. However, if small strains are applied on the GaAs substrate, the InN film could be grown in a zincblende modification, because its lattice mismatch with the substrate is smaller than the GaN case.

In Fig. 4, we show the calculated surface formation energy, $\gamma$, as a function of the difference of the chemical potentials of the monolayer constituents, $\Delta \mu$, at neutral charge states of the systems [11]. From Fig. 4, we note that the nitridation of the GaAs (110) surface is the most important 
Table I. Calculated selected structural parameters for the GaAs/GaN and GaAs/InN (110) surface relaxations as defined in Fig. 1, compared with other theoretical results(the angles $\omega \alpha$ and $\beta$ are in degrees).

\begin{tabular}{|l|l|l|l|l|l|l|}
\hline & $\Delta_{1, \perp}(\AA)$ & $\Delta_{1, x}(\AA)$ & $\Delta_{2, \perp}(\AA)$ & $\omega$ & $\alpha$ & $\beta$ \\
\hline $\mathrm{GaAs} / \mathrm{GaN}$ & 0.002 & 4.905 & 0.13 & 0.21 & 103.7 & 104.9 \\
\hline $\mathrm{GaAs} / \mathrm{InN}$ & 0.140 & 4.682 & 0.12 & 9.6 & 101.6 & 111.5 \\
\hline $\mathrm{GaN}[10]$ & 0.247 & 3.650 & 0.039 & 15.3 & 100.3 & 116.0 \\
\hline $\mathrm{InN}[10]$ & 0.250 & 4.002 & 0.002 & 13.0 & 101.3 & 115.7 \\
\hline
\end{tabular}

step for the initial growth of the III-Nitride, because the adsorption of a $\mathrm{N}$ monolayer is the most stable system, even at the Ga-rich conditions. The same picture is obtained for the InN growth. The main difference between GaN monolayer and the InN monolayer is the value of their surface formation energy. While for the $\mathrm{GaN}$ monolayer, we found $\gamma=$ $213 \mathrm{meV} / \AA^{2}$, for the InN one, we got $\gamma=204 \mathrm{meV} / \AA^{2}$, and both systems are stable only at Ga-rich(In-rich) conditions.

Finally, we presented our results of a systematic theoretical study of the adsorption of $\mathrm{Ga}$, In and $\mathrm{N}$ over GaAs (110) surfaces. Our results show that both the InN and GaN growth over GaAs (110) can take place, and the resulting Nitride film will have hexagonal crystalline symmetry. Also, the GaN growth is energetically more favorable than the $\mathrm{InN}$ growth. However, if we apply small strains on the substrate for the InN growth, we can get cubic samples. A complete description of our obtained results will be published soon elsewhere.

\section{Acknowledgements}

The computer resources were provided by the Centro Nacional de Processamento de Alto Desempenho em Minas Gerais \& Região Centro-Oeste (CENAPAD-MG/CO). A. de Paiva acknowledge the PIBIC-FAPEMIG/UFSJ program for the finantial support.

\section{References}

[1] S. C. Jain, M. Willander, J. Narayan, and R. van Overstraeten, J. Appl. Phys. 87, 965 (2000).

[2] S. A. Ding, G. Neuhold, J. H. Weaver, P. Häberle, K. Horn, O. Brandt, H. Yang, and K. Ploog, J. Vac. Sci. Technol. A14, 819 (1996).
[3] P. Hill, D. I. Westwood, L. Haworth, J. Lu, and J. E. MacDonald, J. Vac. Sci. Technol. B15, 1133 (1997).

[4] D. Troost, H. -U. Baier, A. Berger, and W. Mönch, Surf. Sci. 242, 324 (1991).

[5] X. Gonze, J.-M. Beuken, R. Caracas, F. Detraux, M. Fuchs, G.-M. Rignanese, L. Sindic, M. Verstraete, G. Zerah, F. Jollet, M. Torrent, A. Roy, M. Mikami, Ph. Ghosez, J.-Y. Raty, and D. C. Allan, Comput. Mater. Sci. 25, 478 (2002), and references therein.

[6] C. Hartwigsen, S. Goedecker, and J. Hutter, Phys. Rev. B58, 3641 (1998).

[7] H. J. Monkhorst and J. D. Pack, Phys. Rev. B13, 5188 (1976).

[8] A. de Paiva, J. L. A. Alves, and H. W. Leite Alves, phys. stat. sol. (c) 0, 417 (2002), and references therein.

[9] H. Okumura, H. Hamaguchi, G. Feuillet, Y. Ishida, and S. Yoshida, Appl. Phys. Lett. 72, 3056 (1998).

[10] H. W. Leite Alves, J. L. A. Alves, R. A. Nogueira, and J. R. Leite, Braz. J. Phys. 29, 817 (1999).

[11] The surface formation energy, $\gamma$, was defined as: $\gamma=\frac{1}{2} E_{C}^{\prime}-\frac{1}{2} n_{e} \mu_{e}-\frac{1}{4}\left(N_{X}-N_{N}\right) \Delta \mu$, where $E_{C}^{\prime}$ is the cohesion energy per surface area, $n_{e}$ is the number of electrons transferred from the electron reservoir $\left(n_{e}=0\right.$, in our case), $\Delta \mu$ is the difference of chemical potential of the constituents $\mathrm{N}, \mathrm{Ga}$ or In, and $X$ is the cation $\mathrm{Ga}$ or In. The restriction for $\Delta \mu$ is $-\Delta H \leq \Delta \mu \leq \Delta H$, where $\Delta H$ is the heat of formation of GaAs, $0.74 \mathrm{eV}$ (W. G. Schmidt and F. Bechstedt, Phys. Rev. B54, 16742 (1996)) and, we have followed the recipe developed by J. E. Northrup, Phys. Rev. Lett. 62, 2487 (1989) and by H. W. Leite Alves, Braz. J. Phys. 27/A, 121 (1997). 